\title{
POSITIVE SOLUTIONS FOR A SYSTEM OF SINGULAR SECOND ORDER NONLOCAL BOUNDARY VALUE PROBLEMS
}

\author{
Naseer Ahmad Asif, Paul W. Eloe, and Rahmat Ali Khan \\ Abstract. Sufficient conditions for the existence of positive solutions for \\ a coupled system of nonlinear nonlocal boundary value problems of the \\ type

$$
\begin{aligned}
& -x^{\prime \prime}(t)=f(t, y(t)), \quad t \in(0,1), \\
& -y^{\prime \prime}(t)=g(t, x(t)), \quad t \in(0,1), \\
& x(0)=y(0)=0, x(1)=\alpha x(\eta), y(1)=\alpha y(\eta),
\end{aligned}
$$ \\ are obtained. The nonlinearities $f, g:(0,1) \times(0, \infty) \rightarrow(0, \infty)$ are con- \\ tinuous and may be singular at $t=0, t=1, x=0$, or $y=0$. The \\ parameters $\eta, \alpha$ satisfy $\eta \in(0,1), 0<\alpha<1 / \eta$. An example is provided \\ to illustrate the results.
}

\section{Introduction}

Nonlocal boundary value problems (BVPs) arise in different areas of applied mathematics and physics. For example, the vibration of a guy wire composed of $N$ parts with a uniform cross section and different densities in different parts can be modeled as a nonlocal boundary value problem [18]; problems in the theory of elastic stability can also be modeled as nonlocal boundary value problems [19].

The study of nonlocal BVPs for linear second order ordinary differential equations was initiated by Il'in and Moiseev in $[10,11]$ and extended to nonlocal linear elliptic boundary value problems by Bitsadze and Samarskiǐ, [2, 3, 4]. Existence theory for nonlinear three-point boundary value problems was initiated by Gupta [9]. Since then the study of nonlinear regular multi-point BVPs has attracted the attention of many researchers; see for example, $[5,9,13,14$, $15,17,18,20]$ for scalar equations, and for systems of ordinary differential equations, see $[6,7,12]$.

Received January 1, 2009; Revised June 2, 2009.

2000 Mathematics Subject Classification. 34B16, 34B18.

Key words and phrases. positive solutions, singular system of ordinary differential equations, three-point nonlocal boundary value problem. 
Recently, the study of singular BVPs has also attracted some attention. An excellent resource with an extensive bibliography was produced by Agarwal and O'Regan [1]. Recently, S. Xie and J. Zhu [21] applied topological degree theory in a cone to study the following two point BVP for a coupled system of nonlinear fourth-order ordinary differential equations

$$
\begin{aligned}
-x^{(4)} & =f_{1}(t, y), \quad t \in(0,1), \\
-y^{\prime \prime} & =f_{2}(t, x), \quad t \in(0,1), \\
x(0) & =x(1)=x^{\prime \prime}(0)=x^{\prime \prime}(1)=0, \\
y(0) & =y(1)=0 .
\end{aligned}
$$

In [21], the nonlinearities $f_{i} \in C\left((0,1) \times \mathbb{R}^{+}, \mathbb{R}^{+}\right)$satisfy $f_{i}(t, 0) \equiv 0(i=1,2)$ and may be singular at $t=0$ or $t=1$ only.

More recently, Y. Zhou and Y. Xu [23] studied the following nonlocal BVP for a system of second order regular ordinary differential equations

$$
\begin{gathered}
-x^{\prime \prime}(t)=f(t, y), \quad t \in(0,1), \\
-y^{\prime \prime}(t)=g(t, x), \quad t \in(0,1), \\
x(0)=0, \quad x(1)=\alpha x(\eta), \\
y(0)=0, \quad y(1)=\alpha y(\eta),
\end{gathered}
$$

where $\eta \in(0,1), 0<\alpha<1 / \eta, f, g \in C([0,1] \times[0, \infty),[0, \infty)), f(t, 0) \equiv 0$, $g(t, 0) \equiv 0$. The above system was extended to the singular case by B. Liu, $\mathrm{L}$. Liu, and $\mathrm{Y}$. Wu [16], where the nonlinearities $f, g$ were assumed to be singular at $t=0$ or $t=1$ together with the assumption that $f(t, 0) \equiv 0, g(t, 0) \equiv 0$, $t \in(0,1)$.

In this paper, we generalize the system (1.2) by allowing $f, g$ to be singular at $t=0, t=1, x=0$, or $y=0$ and obtain sufficient conditions for the existence of a positive solution of the BVP for the system of singular equations, (1.2). By singularity we mean that the functions $f(t, u)$ or $g(t, u)$ are allowed to be unbounded at $t=0, t=1$, or $u=0$. In general, the assumption that there exist singularities with respect to the dependent variable is not new; see $[1,6]$, for example. However, in the case of nonlocal boundary conditions and coupled systems of ordinary differential equations, we believe this assumption is new.

Throughout this paper, we shall assume that

$$
f, g:(0,1) \times(0, \infty) \rightarrow(0, \infty)
$$

are continuous and may be singular at $t=0, t=1$, or $u=0$. We also assume that $f(t, 0), g(t, 0)$ are not identically 0 . Let $N>\max \left\{\frac{1}{\eta}, \frac{1}{1-\eta}, \frac{2-\alpha}{1-\alpha \eta}\right\}$ denote a fixed positive integer. Assume that the following conditions hold:

$\left(A_{1}\right)$ there exist $K, L \in C((0,1),(0, \infty))$ and $F, G \in C((0, \infty),(0, \infty))$ such that

$$
f(t, u) \leq K(t) F(u), \quad g(t, u) \leq L(t) G(u), \quad t \in(0,1), u \in(0, \infty)
$$


and

$a:=\int_{0}^{1} t(1-t) K(t) d t<+\infty, \quad b:=\int_{0}^{1} t(1-t) L(t) d t<+\infty ;$

$\left(A_{2}\right)$ there exist $\alpha_{1}, \alpha_{2} \in(0, \infty)$ with $\alpha_{1} \alpha_{2} \leq 1$ such that

$$
\lim _{u \rightarrow \infty} \frac{F(u)}{u^{\alpha_{1}}} \rightarrow 0, \quad \lim _{u \rightarrow \infty} \frac{G(u)}{u^{\alpha_{2}}} \rightarrow 0
$$

$\left(A_{3}\right)$ there exist $\beta_{1}, \beta_{2} \in(0, \infty)$ with $\beta_{1} \beta_{2} \geq 1$ such that

$$
\liminf _{u \rightarrow 0^{+}} \min _{t \in[\eta, 1]} \frac{f(t, u)}{u^{\beta_{1}}}>0, \quad \liminf _{u \rightarrow 0^{+}} \min _{t \in[\eta, 1]} \frac{g(t, u)}{u^{\beta_{2}}}>0 ;
$$

$\left(A_{4}\right) f(t, u), G(u)$ are non-increasing with respect to $u$ and for each fixed $n \in\{N, N+1, N+2, \ldots\}$, there exists a constant $M_{1}>0$ such that $t \in\left[\frac{1}{n}, 1-\frac{1}{n}\right]$,

$$
f\left(t, \frac{1}{n}+b \mu_{n} G\left(\frac{1}{n}\right)\right) \geq M_{1}\left(\nu_{n} \int_{\eta}^{1-1 / n}\left(s-\frac{1}{n}\right)\left(1-\frac{1}{n}-s\right) d s\right)^{-1} ;
$$

$\left(A_{5}\right) F(u), g(t, u)$ are non-increasing with respect to $u$ and for each fixed $n \in\{N, N+1, N+2, \ldots\}$, there exists a constant $M_{2}>0$ such that

$$
F\left(\nu_{n} \int_{\eta}^{1-1 / n}\left(s-\frac{1}{n}\right)\left(1-\frac{1}{n}-s\right) g\left(s, M_{2}\right) d s\right) \leq \frac{M_{2}-\frac{1}{n}}{a \mu_{n}} .
$$

The parameters $\mu_{n}$ and $\nu_{n}$ in $\left(A_{4}\right)$ and $\left(A_{5}\right)$ are given by

$$
\mu_{n}=\frac{\max \{1, \alpha\}}{1-\frac{2}{n}+\frac{\alpha}{n}-\alpha \eta}, \quad \nu_{n}=\frac{\min \{1, \alpha\} \min \left\{\eta-\frac{1}{n}, 1-\frac{1}{n}-\eta\right\}}{1-\frac{2}{n}+\frac{\alpha}{n}-\alpha \eta} .
$$

Since $N>\max \left\{\frac{1}{\eta}, \frac{1}{1-\eta}, \frac{2-\alpha}{1-\alpha \eta}\right\}, \mu_{n}, \nu_{n}>0$.

We state the main results of this paper here.

Theorem 1.1. Assume that $\left(A_{1}\right)-\left(A_{3}\right)$ hold. Then the system (1.1) has at least one positive solution.

Theorem 1.2. Assume that $\left(A_{1}\right),\left(A_{2}\right)$ and $\left(A_{4}\right)$ hold. Then the system (1.1) has at least one positive solution.

Theorem 1.3. Assume that $\left(A_{1}\right),\left(A_{3}\right)$ and $\left(A_{5}\right)$ hold. Then the system (1.1) has at least one positive solution.

Theorem 1.4. Assume that $\left(A_{1}\right),\left(A_{4}\right)$ and $\left(A_{5}\right)$ hold. Then the system (1.1) has at least one positive solution. 


\section{Preliminaries}

For each $x \in C[0,1]$ we write $\|x\|=\max \{|x(t)|: t \in[0,1]\}$. Clearly, $C[0,1]$ with the norm $\|\cdot\|$ is a Banach space. For $n \geq N$, define a cone $P$, and a cone $K_{n}$ of $C\left[\frac{1}{n}, 1-\frac{1}{n}\right]$ as follows:

$$
\begin{aligned}
P & =\{x \in C[0,1]: x(t) \geq 0, t \in[0,1]\}, \\
P_{n} & =\left\{x \in P: x \text { is concave on }[0,1], \min _{t \in\left[\frac{1}{n}, 1-\frac{1}{n}\right]} x(t) \geq \frac{1}{n}\right\}, \\
K_{n} & =\left\{x \in C\left[\frac{1}{n}, 1-\frac{1}{n}\right]: x \text { is concave on }[0,1]\right\} .
\end{aligned}
$$

For any real constant $r>0$, define

$$
\Omega_{r}=\{x \in C[0,1]:\|x\|<r\}
$$

as an open neighborhood of $0 \in C[0,1]$ of radius $r .(x(t), y(t))$ is called a positive solution of (1.1) if

$$
(x, y) \in\left(C[0,1] \cap C^{2}(0,1)\right) \times\left(C[0,1] \cap C^{2}(0,1)\right),
$$

$x(t)>0, y(t)>0$ on $(0,1)$ and $(x, y)$ satisfies $(1.1)$.

The proofs of our main results (Theorems 1.1-1.4) are based on the GuoKrasnosel'skii fixed-point theorem.

Lemma 2.1 ([8, Guo Krasnosel'skii Fixed-Point Theorem]). Let $K$ be a cone of a real Banach space $E$, and let $\Omega_{1}, \Omega_{2}$ be bounded open neighborhoods of $0 \in E$, and assume $\Omega_{1} \subset \Omega_{2}$. Suppose that $T: K \cap\left(\bar{\Omega}_{2} \backslash \Omega_{1}\right) \rightarrow K$ is completely continuous such that one of the following conditions holds:

(i) $\|T x\| \leq\|x\|$ for $x \in \partial \Omega_{1} \cap K ;\|T x\| \geq\|x\|$ for $x \in \partial \Omega_{2} \cap K$;

(ii) $\|T x\| \leq\|x\|$ for $x \in \partial \Omega_{2} \cap K ;\|T x\| \geq\|x\|$ for $x \in \partial \Omega_{1} \cap K$.

Then, $T$ has a fixed point in $K \cap\left(\bar{\Omega}_{2} \backslash \Omega_{1}\right)$.

For fixed $n \geq N$ and $z \in C[0,1]$, the linear boundary value problem

$$
\begin{aligned}
-u^{\prime \prime}(t) & =z(t), \quad t \in\left[\frac{1}{n}, 1-\frac{1}{n}\right], \\
u\left(\frac{1}{n}\right) & =\frac{1}{n}, \quad u\left(1-\frac{1}{n}\right)=\alpha u(\eta)+\frac{1-\alpha}{n},
\end{aligned}
$$

has a unique solution

$$
u(t)=\frac{1}{n}+\int_{1 / n}^{1-1 / n} H_{n}(t, s) z(s) d s,
$$


where $H_{n}:\left[\frac{1}{n}, 1-\frac{1}{n}\right] \times\left[\frac{1}{n}, 1-\frac{1}{n}\right] \rightarrow[0, \infty)$ is an associated Green's function and is defined by

$$
H_{n}(t, s)= \begin{cases}\frac{\left(t-\frac{1}{n}\right)\left(\left(1-\frac{1}{n}-s\right)-\alpha(\eta-s)\right)}{1-\frac{2}{n}+\frac{\alpha}{n}-\alpha \eta}-(t-s), & \frac{1}{n} \leq s \leq t \leq 1-\frac{1}{n}, s \leq \eta, \\ \frac{\left(t-\frac{1}{n}\right)\left(\left(1-\frac{1}{n}-s\right)-\alpha(\eta-s)\right)}{1-\frac{2}{n}+\frac{\alpha}{n}-\alpha \eta}, & \frac{1}{n} \leq t \leq s \leq 1-\frac{1}{n}, s \leq \eta, \\ \frac{\left(t-\frac{1}{n}\right)\left(1-\frac{1}{n}-s\right)}{1-\frac{2}{n}+\frac{\alpha}{n}-\alpha \eta}, & \frac{1}{n} \leq t \leq s \leq 1-\frac{1}{n}, s \geq \eta, \\ \frac{\left(t-\frac{1}{n}\right)\left(1-\frac{1}{n}-s\right)}{1-\frac{2}{n}+\frac{\alpha}{n}-\alpha \eta}-(t-s), & \frac{1}{n} \leq s \leq t \leq 1-\frac{1}{n}, s \geq \eta .\end{cases}
$$

We note that $H_{n}(t, s) \rightarrow H(t, s)$ as $n \rightarrow \infty$, where

$$
H(t, s)= \begin{cases}\frac{t(1-s)}{1-\alpha \eta}-\frac{\alpha t(\eta-s)}{1-\alpha \eta}-(t-s), & 0 \leq s \leq t \leq 1, s \leq \eta, \\ \frac{t(1-s)}{1-\alpha \eta}-\frac{\alpha t(\eta-s)}{1-\alpha \eta}, & 0 \leq t \leq s \leq 1, s \leq \eta, \\ \frac{t(1-s)}{1-\alpha \eta}, & 0 \leq t \leq s \leq 1, s \geq \eta, \\ \frac{t(1-s)}{1-\alpha \eta}-(t-s), & 0 \leq s \leq t \leq 1-, s \geq \eta,\end{cases}
$$

is the Green's function corresponding the boundary value problem

$$
\begin{aligned}
& -u^{\prime \prime}(t)=z(t), \quad t \in[0,1], \\
& u(0)=0, \quad u(1)=\alpha u(\eta)
\end{aligned}
$$

with

$$
u(t)=\int_{0}^{1} H(t, s) z(s) d s,
$$

as its integral representation. We need the following properties of the Green's function $H_{n}$ in the sequel. For the proof, see [22].

Lemma 2.2. The function $H_{n}$ can be written as

$$
H_{n}(t, s)=G_{n}(t, s)+\frac{\alpha\left(t-\frac{1}{n}\right)}{1-\frac{2}{n}+\frac{\alpha}{n}-\alpha \eta} G_{n}(\eta, s),
$$

where

$$
G_{n}(t, s)=\frac{n}{n-2} \begin{cases}\left(s-\frac{1}{n}\right)\left(1-\frac{1}{n}-t\right), & \frac{1}{n} \leq s \leq t \leq 1-\frac{1}{n} \\ \left(t-\frac{1}{n}\right)\left(1-\frac{1}{n}-s\right), & \frac{1}{n} \leq t \leq s \leq 1-\frac{1}{n}\end{cases}
$$


Lemma 2.3. Let

$$
\mu_{n}=\frac{\max \{1, \alpha\}}{1-\frac{2}{n}+\frac{\alpha}{n}-\alpha \eta}, \quad \nu_{n}=\frac{\min \{1, \alpha\} \min \left\{\eta-\frac{1}{n}, 1-\frac{1}{n}-\eta\right\}}{1-\frac{2}{n}+\frac{\alpha}{n}-\alpha \eta} .
$$

Then

(i) $H_{n}(t, s) \leq \mu_{n}\left(s-\frac{1}{n}\right)\left(1-\frac{1}{n}-s\right), \quad(t, s) \in\left[\frac{1}{n}, 1-\frac{1}{n}\right] \times\left[\frac{1}{n}, 1-\frac{1}{n}\right]$,

(ii) $H_{n}(t, s) \geq \nu_{n}\left(s-\frac{1}{n}\right)\left(1-\frac{1}{n}-s\right), \quad(t, s) \in\left[\eta, 1-\frac{1}{n}\right] \times\left[\frac{1}{n}, 1-\frac{1}{n}\right]$.

Now consider the system of nonlinear non-singular BVPs

$$
\begin{aligned}
-x^{\prime \prime}(t) & =f\left(t, \max \left\{\frac{1}{n}, y(t)\right\}\right), \quad t \in\left[\frac{1}{n}, 1-\frac{1}{n}\right], \\
-y^{\prime \prime}(t) & =g\left(t, \max \left\{\frac{1}{n}, x(t)\right\}\right), \quad t \in\left[\frac{1}{n}, 1-\frac{1}{n}\right], \\
x\left(\frac{1}{n}\right) & =\frac{1}{n}, \quad x\left(1-\frac{1}{n}\right)=\alpha x(\eta)+\frac{1-\alpha}{n}, \\
y\left(\frac{1}{n}\right) & =\frac{1}{n}, \quad y\left(1-\frac{1}{n}\right)=\alpha y(\eta)+\frac{1-\alpha}{n},
\end{aligned}
$$

where $n>N$. Write (2.6) as an equivalent system of integral equations

$$
\begin{aligned}
& x(t)=\frac{1}{n}+\int_{1 / n}^{1-1 / n} H_{n}(t, s) f\left(s, \max \left\{\frac{1}{n}, y(s)\right\}\right) d s, \\
& y(t)=\frac{1}{n}+\int_{1 / n}^{1-1 / n} H_{n}(t, s) g\left(s, \max \left\{\frac{1}{n}, x(s)\right\}\right) d s .
\end{aligned}
$$

Thus, $(x, y)$ is a solution of (2.6) if and only if

$$
(x, y) \in C\left[\frac{1}{n}, 1-\frac{1}{n}\right] \times C\left[\frac{1}{n}, 1-\frac{1}{n}\right]
$$

and $(x, y)$ is a solution of $(2.7)$.

Define operators $A_{n}, B_{n}, T_{n}: K_{n} \rightarrow K_{n}$ by

$$
\begin{aligned}
& \left(A_{n} y\right)(t)=\frac{1}{n}+\int_{1 / n}^{1-1 / n} H_{n}(t, s) f\left(s, \max \left\{\frac{1}{n}, y(s)\right\}\right) d s \\
& \left(B_{n} x\right)(t)=\frac{1}{n}+\int_{1 / n}^{1-1 / n} H_{n}(t, s) g\left(s, \max \left\{\frac{1}{n}, x(s)\right\}\right) d s \\
& \left(T_{n} x\right)(t)=\left(A_{n}\left(B_{n} x\right)\right)(t) .
\end{aligned}
$$

If $u_{n} \in K_{n}$ is a fixed point of $T_{n}$, then the system of BVPs (2.6) has a solution $\left(x_{n}, y_{n}\right)$ given by

$$
\left\{\begin{array}{l}
x_{n}(t)=u_{n}(t) \\
y_{n}(t)=\left(B_{n} u_{n}\right)(t) .
\end{array}\right.
$$

By construction, the system of BVPs (2.6) is regular and so the following lemma is standard.

Lemma 2.4. Assume $f, g:(0,1) \times(0, \infty) \rightarrow[0, \infty)$ are continuous. Then $T_{n}: K_{n} \rightarrow K_{n}$ is completely continuous. 


\section{Main results}

Proof of Theorem 1.1. By $\left(A_{2}\right)$, there exist constants $C_{1}, C_{2}, N_{1}, N_{2}>0$ such that

$$
4^{\alpha_{1}} a b^{\alpha_{1}} \mu_{n}^{\alpha_{1}+1} C_{1} C_{2}^{\alpha_{1}}<1
$$

and

$$
F(x) \leq C_{1} x^{\alpha_{1}}+N_{1}, \quad G(x) \leq C_{2} x^{\alpha_{2}}+N_{2} \text { for } x \geq \frac{1}{n} .
$$

Choose a constant $R>0$ such that

$$
R \geq \frac{\frac{1}{n}+\frac{2^{\alpha_{1}} a \mu_{n} C_{1}}{n^{\alpha_{1}}}+a \mu_{n} N_{1}+4^{\alpha_{1}} a b^{\alpha_{1}} \mu_{n}^{\alpha_{1}+1} C_{1} N_{2}^{\alpha_{1}}}{1-4^{\alpha_{1}} a b^{\alpha_{1}} \mu_{n}^{\alpha_{1}+1} C_{1} C_{2}^{\alpha_{1}}} .
$$

For any $u \in \partial \Omega_{R} \cap K_{n}$, using (2.8) and $\left(A_{1}\right)$, we have

$$
\begin{aligned}
\left(T_{n} u\right)(t) & =\left(A_{n}\left(B_{n} u\right)\right)(t)=\frac{1}{n}+\int_{1 / n}^{1-1 / n} H_{n}(t, s) f\left(s,\left(B_{n} u\right)(s)\right) d s \\
& =\frac{1}{n}+\int_{1 / n}^{1-1 / n} H_{n}(t, s) f\left(s, \frac{1}{n}+\int_{1 / n}^{1-1 / n} H_{n}(s, \tau) g(\tau, u(\tau)) d \tau\right) d s \\
& \leq \frac{1}{n}+\int_{1 / n}^{1-1 / n} H_{n}(t, s) K(s) F\left(\frac{1}{n}+\int_{1 / n}^{1-1 / n} H_{n}(s, \tau) g(\tau, u(\tau)) d \tau\right) d s .
\end{aligned}
$$

In view of $(3.2)$ and $\left(A_{2}\right)$, it follows that

$$
\begin{aligned}
& \left(T_{n} u\right)(t) \\
\leq & \frac{1}{n}+\int_{1 / n}^{1-1 / n} H_{n}(t, s) K(s)\left(C_{1}\left(\frac{1}{n}+\int_{1 / n}^{1-1 / n} H_{n}(s, \tau) g(\tau, u(\tau)) d \tau\right)^{\alpha_{1}}+N_{1}\right) d s \\
= & \frac{1}{n}+C_{1} \int_{1 / n}^{1-1 / n} H_{n}(t, s) K(s)\left(\frac{1}{n}+\int_{1 / n}^{1-1 / n} H_{n}(s, \tau) g(\tau, u(\tau)) d \tau\right)^{\alpha_{1}} d s \\
& +N_{1} \int_{1 / n}^{1-1 / n} H_{n}(t, s) K(s) d s \\
\leq & \frac{1}{n}+C_{1} \int_{1 / n}^{1-1 / n} H_{n}(t, s) K(s)\left(\frac{1}{n}+\int_{1 / n}^{1-1 / n} H_{n}(s, \tau) L(\tau) G(u(\tau)) d \tau\right)^{\alpha_{1}} d s \\
& +N_{1} \int_{1 / n}^{1-1 / n} H_{n}(t, s) K(s) d s \\
\leq & \frac{1}{n}+C_{1} \int_{1 / n}^{1-1 / n} H_{n}(t, s) K(s) \\
& \cdot\left(\frac{1}{n}+\int_{1 / n}^{1-1 / n} H_{n}(s, \tau) L(\tau)\left(C_{2}(u(\tau))^{\alpha_{2}}+N_{2}\right) d \tau\right)^{\alpha_{1}} d s
\end{aligned}
$$




$$
+N_{1} \int_{1 / n}^{1-1 / n} H_{n}(t, s) K(s) d s .
$$

Employing (i) of Lemma 2.3, we obtain

$$
\begin{aligned}
& \left(T_{n} u\right)(t) \\
\leq & \frac{1}{n}+C_{1} \mu_{n} \int_{1 / n}^{1-1 / n}\left(s-\frac{1}{n}\right)\left(1-\frac{1}{n}-s\right) K(s) d s \\
& \cdot\left(\frac{1}{n}+\mu_{n} \int_{1 / n}^{1-1 / n}\left(\tau-\frac{1}{n}\right)\left(1-\frac{1}{n}-\tau\right) L(\tau)\left(C_{2}(u(\tau))^{\alpha_{2}}+N_{2}\right) d \tau\right)^{\alpha_{1}} \\
& +N_{1} \mu_{n} \int_{1 / n}^{1-1 / n}\left(s-\frac{1}{n}\right)\left(1-\frac{1}{n}-s\right) K(s) d s \\
\leq & \frac{1}{n}+C_{1} \mu_{n} \int_{1 / n}^{1-1 / n} s(1-s) K(s) d s \\
& \cdot\left(\frac{1}{n}+\mu_{n} \int_{1 / n}^{1-1 / n} \tau(1-\tau) L(\tau)\left(C_{2}(u(\tau))^{\alpha_{2}}+N_{2}\right) d \tau\right)^{\alpha_{1}} \\
& +N_{1} \mu_{n} \int_{1 / n}^{1-1 / n} s(1-s) K(s) d s .
\end{aligned}
$$

Hence,

$$
\begin{aligned}
& \left(T_{n} u\right)(t) \\
\leq & \frac{1}{n}+C_{1} \mu_{n} \int_{1 / n}^{1-1 / n} s(1-s) K(s) d s \\
& \cdot\left(\frac{1}{n}+\mu_{n} \int_{1 / n}^{1-1 / n} \tau(1-\tau) L(\tau)\left(C_{2}\|u\|^{\alpha_{2}}+N_{2}\right) d \tau\right)^{\alpha_{1}} \\
& +N_{1} \mu_{n} \int_{1 / n}^{1-1 / n} s(1-s) K(s) d s \\
\leq & \frac{1}{n}+\mu_{n} C_{1} \int_{0}^{1} s(1-s) K(s) d s \\
& \cdot\left(\frac{1}{n}+\mu_{n} \int_{0}^{1} \tau(1-\tau) L(\tau) d \tau\left(C_{2}\|u\|^{\alpha_{2}}+N_{2}\right)\right)^{\alpha_{1}} \\
& +\mu_{n} N_{1} \int_{0}^{1} s(1-s) K(s) d s \\
\leq & \frac{1}{n}+a \mu_{n} N_{1}+2^{\alpha_{1}} a \mu_{n} C_{1}\left(\frac{1}{n^{\alpha_{1}}}+b^{\alpha_{1}} \mu_{n}^{\alpha_{1}}\left(C_{2}\|u\|^{\alpha_{2}}+N_{2}\right)^{\alpha_{1}}\right) \\
\leq & \frac{1}{n}+\frac{2^{\alpha_{1}} a \mu_{n} C_{1}}{n^{\alpha_{1}}}+a \mu_{n} N_{1}+2^{2 \alpha_{1}} a b^{\alpha_{1}} \mu_{n}^{\alpha_{1}+1} C_{1}\left(C_{2}^{\alpha_{1}}\|u\|^{\alpha_{1} \alpha_{2}}+N_{2}^{\alpha_{1}}\right) .
\end{aligned}
$$


Using (3.3), we obtain

$$
\left\|T_{n} u\right\| \leq\|u\| \text { for all } u \in \partial \Omega_{R} \cap K_{n} .
$$

Now, by $\left(A_{3}\right)$, there exist constants $C_{3}, C_{4}>0$ and $\rho \in(0, R)$ such that

$$
f(t, x) \geq C_{3} x^{\beta_{1}}, g(t, x) \geq C_{4} x^{\beta_{2}} \quad \text { for } x \in[0, \rho] \text { and } t \in[\eta, 1] .
$$

Choose

$$
r_{n}=\min \left\{\rho, \frac{C_{3} C_{4}^{\beta_{1}} \nu_{n}^{\beta_{1}+1}}{n^{\beta_{1} \beta_{2}}}\left(\int_{\eta}^{1-1 / n}\left(s-\frac{1}{n}\right)\left(1-\frac{1}{n}-s\right) d s\right)^{\beta_{1}+1}\right\} .
$$

For any $u \in \partial \Omega_{r_{n}} \cap K_{n}$, using (2.8), (3.5) and (ii) of Lemma 2.3, we have

$$
\begin{aligned}
& \left(T_{n} u\right)(t)=\left(A_{n}\left(B_{n} u\right)\right)(t) \\
& =\frac{1}{n}+\int_{1 / n}^{1-1 / n} H_{n}(t, s) f\left(s, \frac{1}{n}+\int_{1 / n}^{1-1 / n} H_{n}(s, \tau) g(\tau, u(\tau)) d \tau\right) d s \\
& \geq \int_{1 / n}^{1-1 / n} H_{n}(t, s) f\left(s, \frac{1}{n}+\int_{1 / n}^{1-1 / n} H_{n}(s, \tau) g(\tau, u(\tau)) d \tau\right) d s \\
& \geq \int_{\eta}^{1-1 / n} H_{n}(t, s) f\left(s, \frac{1}{n}+\int_{1 / n}^{1-1 / n} H_{n}(s, \tau) g(\tau, u(\tau)) d \tau\right) d s \\
& \geq C_{3} \int_{\eta}^{1-1 / n} H_{n}(t, s)\left(\int_{\eta}^{1-1 / n} H_{n}(s, \tau) g(\tau, u(\tau)) d \tau\right)^{\beta_{1}} d s \\
& \geq C_{3} \nu_{n} \int_{\eta}^{1-1 / n}\left(s-\frac{1}{n}\right)\left(1-\frac{1}{n}-s\right) d s \\
& \cdot\left(\nu_{n} \int_{\eta}^{1-1 / n}\left(\tau-\frac{1}{n}\right)\left(1-\frac{1}{n}-\tau\right) g(\tau, u(\tau)) d \tau\right)^{\beta_{1}} \\
& \geq C_{3} \nu_{n}^{\beta_{1}+1} \int_{\eta}^{1-1 / n}\left(s-\frac{1}{n}\right)\left(1-\frac{1}{n}-s\right) d s \\
& \cdot\left(C_{4} \int_{\eta}^{1-1 / n}\left(\tau-\frac{1}{n}\right)\left(1-\frac{1}{n}-\tau\right)(u(\tau))^{\beta_{2}} d \tau\right)^{\beta_{1}} \\
& \geq \frac{C_{3} C_{4}^{\beta_{1}} \nu_{n}^{\beta_{1}+1}}{n^{\beta_{1} \beta_{2}}}\left(\int_{\eta}^{1-1 / n}\left(s-\frac{1}{n}\right)\left(1-\frac{1}{n}-s\right) d s\right)^{\beta_{1}+1} .
\end{aligned}
$$

Thus, in view of (3.6), it follows that

$$
\left\|T_{n} u\right\| \geq\|u\| \text { for } u \in \partial \Omega_{r_{n}} \cap K_{n} .
$$

By Lemma 2.1, $T_{n}$ has a fixed point $u_{n} \in K_{n} \cap\left(\bar{\Omega}_{R} \backslash \Omega_{r_{n}}\right)$. Note that

$$
r_{n} \leq u_{n}(t) \leq R \text { for all } t \in\left[\frac{1}{n}, 1-\frac{1}{n}\right]
$$


and $r_{n} \rightarrow 0$ as $n \rightarrow \infty$. Thus, we have exhibited a uniform bound for each $u_{n} \in\left[\frac{1}{n}, 1-\frac{1}{n}\right]$ and for $m \geq n,\left\{u_{m}\right\}$ is uniformly bounded on $\left[\frac{1}{n}, 1-\frac{1}{n}\right]$.

To show that $\left\{u_{m}\right\}$ for $m \geq n$, is equicontinuous on $\left[\frac{1}{n}, 1-\frac{1}{n}\right]$, consider for $t \in\left[\frac{1}{n}, 1-\frac{1}{n}\right]$, the integral equation

$$
u_{m}(t)=u_{m}\left(\frac{1}{m}\right)+\int_{1 / m}^{1-1 / m} H_{m}(t, s) f\left(s,\left(B_{m} u_{m}\right)(s)\right) d s .
$$

Employ Lemma 2.2 to obtain

$$
\begin{aligned}
& u_{m}(t) \\
= & u_{m}\left(\frac{1}{m}\right)+\int_{1 / m}^{1-1 / m}\left[G_{m}(t, s)+\frac{\alpha\left(t-\frac{1}{m}\right)}{1-\frac{2}{m}+\frac{\alpha}{m}-\alpha \eta} G_{m}(\eta, s)\right] f\left(s,\left(B_{m} u_{m}\right)(s)\right) d s \\
= & u_{m}\left(\frac{1}{m}\right)+\frac{m}{m-2} \int_{1 / m}^{t}\left(s-\frac{1}{m}\right)\left(1-\frac{1}{m}-t\right) f\left(s,\left(B_{m} u_{m}\right)(s)\right) d s \\
& +\frac{m}{m-2} \int_{t}^{1-1 / m}\left(t-\frac{1}{m}\right)\left(1-\frac{1}{m}-s\right) f\left(s,\left(B_{m} u_{m}\right)(s)\right) d s \\
& +\frac{\alpha\left(t-\frac{1}{m}\right)}{1-\frac{2}{m}+\frac{\alpha}{m}-\alpha \eta} \int_{1 / m}^{1-1 / m} G_{m}(\eta, s) f\left(s,\left(B_{m} u_{m}\right)(s)\right) d s .
\end{aligned}
$$

Differentiate with respect to $t$ to obtain

$$
\begin{aligned}
u_{m}^{\prime}(t)= & -\frac{m}{m-2} \int_{1 / m}^{t}\left(s-\frac{1}{m}\right) f\left(s,\left(B_{m} u_{m}\right)(s)\right) d s \\
& +\frac{m}{m-2} \int_{t}^{1-1 / m}\left(1-\frac{1}{m}-s\right) f\left(s,\left(B_{m} u_{m}\right)(s)\right) d s \\
& +\frac{\alpha}{1-\frac{2}{m}+\frac{\alpha}{m}-\alpha \eta} \int_{1 / m}^{1-1 / m} G_{m}(\eta, s) f\left(s,\left(B_{m} u_{m}\right)(s)\right) d s,
\end{aligned}
$$

which implies that for $t \in\left[\frac{1}{n}, 1-\frac{1}{n}\right]$

$$
\begin{aligned}
\left|u_{m}^{\prime}(t)\right| \leq & \int_{1 / m}^{1-1 / m} f\left(s,\left(B_{m} u_{m}\right)(s)\right) d s \\
& +\frac{\alpha}{1-\frac{2}{m}+\frac{\alpha}{m}-\alpha \eta} \int_{1 / m}^{1-1 / m} G_{m}(\eta, s) f\left(s,\left(B_{m} u_{m}\right)(s)\right) d s .
\end{aligned}
$$

Hence, for $m \geq n,\left\{u_{m}\right\}$ is equicontinuous on $\left[\frac{1}{n}, 1-\frac{1}{n}\right]$.

For $m \geq n$, define

$$
v_{m}=\left\{\begin{array}{l}
u_{m}\left(\frac{1}{n}\right), \text { if } 0 \leq t \leq \frac{1}{n} \\
u_{m}(t), \text { if } \frac{1}{n} \leq t \leq 1-\frac{1}{n} \\
\alpha u_{m}(\eta), \text { if } 1-\frac{1}{n} \leq t \leq 1 .
\end{array}\right.
$$


Since $v_{m}$ is a constant extension of $u_{m}$ to $[0,1]$, the sequence $\left\{v_{m}\right\}$ is uniformly bounded and equicontinuous on $[0,1]$. Thus, there exists a subsequence $\left\{v_{n_{k}}\right\}$ of $\left\{v_{m}\right\}$ converging uniformly on $[0,1]$ to $v \in P \cap\left(\bar{\Omega}_{R} \backslash \Omega_{r}\right)$.

We introduce the notation

$$
\begin{aligned}
x_{n_{k}}(t) & =v_{n_{k}}(t), \quad y_{n_{k}}(t)=\frac{1}{n_{k}}+\int_{1 / n_{k}}^{1-1 / n_{k}} H_{n_{k}}(t, s) g\left(s, v_{n_{k}}(s)\right) d s, \\
\bar{x}(t) & =\lim _{n_{k} \rightarrow \infty} x_{n_{k}}(t), \quad \bar{y}(t)=\lim _{n_{k} \rightarrow \infty} y_{n_{k}}(t),
\end{aligned}
$$

and for $t \in[0,1]$ consider the integral equation

$$
x_{n_{k}}(t)=x_{n_{k}}\left(\frac{1}{n_{k}}\right)+\int_{1 / n_{k}}^{1-1 / n_{k}} H_{n_{k}}(t, s) f\left(t, y_{n_{k}}(s)\right) d s .
$$

Letting $n_{k} \rightarrow \infty$, we have

$$
\bar{x}(t)=\bar{x}(0)+\int_{0}^{1} H(t, s) f(t, \bar{y}(s)) d s,
$$

and

$$
\bar{y}(t)=\int_{0}^{1} H(t, s) g(s, \bar{x}(s)) d s, t \in[0,1]
$$

Moreover,

$$
\bar{x}(0)=0, \quad x(1)=\alpha \bar{x}(\eta), \quad \bar{y}(0)=0, \quad \bar{y}(1)=\alpha \bar{y}(\eta) .
$$

Hence, $(\bar{x}(t), \bar{y}(t))$ is a solution of the system (1.2).

Since

$$
f, g:(0,1) \times(0, \infty) \rightarrow(0, \infty),
$$

$f(t, 0), g(t, 0)$ are not identically 0 , and $H$ is of fixed sign on $(0,1) \times(0,1)$, it follows that $\bar{x}, \bar{y}>0$ on $(0,1)$.

Example 3.1. Let

$$
f(t, y)=\frac{1}{t(1-t)}\left(\frac{1}{y}+3 y^{1 / 3}\right), \quad g(t, x)=\frac{1}{t(1-t)}\left(\frac{1}{x}+4 x\right)
$$

and $\alpha=2, \eta=\frac{1}{3}$. Choose

$$
K(t)=L(t)=\frac{1}{t(1-t)}, \quad F(y)=\frac{1}{y}+3 y^{1 / 3}, \quad G(x)=\frac{1}{x}+4 x,
$$

and $\alpha_{1}=\frac{1}{2}, \alpha_{2}=2, \beta_{1}=\beta_{2}=1$. Then $\left(A_{1}\right)-\left(A_{3}\right)$ are satisfied. Hence, by Theorem 1.1, system (1.2) has a positive solution. 
Proof of Theorem 1.2. For $u \in \partial \Omega_{M_{1}} \cap K_{n}$, using (2.8), we obtain for $t \in$ $\left[\frac{1}{n}, 1-\frac{1}{n}\right]$

$$
\begin{aligned}
\left(T_{n} u\right)(t) & =\left(A_{n}\left(B_{n} u\right)\right)(t)=\frac{1}{n}+\int_{1 / n}^{1-1 / n} H_{n}(t, s) f\left(s,\left(B_{n} u\right)(s)\right) d s \\
& =\frac{1}{n}+\int_{1 / n}^{1-1 / n} H_{n}(t, s) f\left(s, \frac{1}{n}+\int_{1 / n}^{1-1 / n} H_{n}(s, \tau) g(\tau, u(\tau)) d \tau\right) d s \\
& \geq \int_{1 / n}^{1-1 / n} H_{n}(t, s) f\left(s, \frac{1}{n}+\int_{1 / n}^{1-1 / n} H_{n}(s, \tau) g(\tau, u(\tau)) d \tau\right) d s .
\end{aligned}
$$

Using $\left(A_{1}\right),\left(A_{4}\right)$ and Lemma 2.3 , we have

$$
\begin{aligned}
& \left(T_{n} u\right)(t) \\
\geq & \int_{1 / n}^{1-1 / n} H_{n}(t, s) f\left(s, \frac{1}{n}+\mu_{n} \int_{1 / n}^{1-1 / n}\left(\tau-\frac{1}{n}\right)\left(1-\frac{1}{n}-\tau\right) g(\tau, u(\tau)) d \tau\right) d s \\
\geq & \int_{1 / n}^{1-1 / n} H_{n}(t, s) f\left(s, \frac{1}{n}+\mu_{n} \int_{1 / n}^{1-1 / n}\left(\tau-\frac{1}{n}\right)\left(1-\frac{1}{n}-\tau\right) L(\tau) G(u(\tau)) d \tau\right) d s \\
\geq & \int_{1 / n}^{1-1 / n} H_{n}(t, s) f\left(s, \frac{1}{n}+\mu_{n} G\left(\frac{1}{n}\right) \int_{1 / n}^{1-1 / n}\left(\tau-\frac{1}{n}\right)\left(1-\frac{1}{n}-\tau\right) L(\tau) d \tau\right) d s \\
\geq & \int_{1 / n}^{1-1 / n} H_{n}(t, s) f\left(s, \frac{1}{n}+b \mu_{n} G\left(\frac{1}{n}\right)\right) d s \\
\geq & M_{1} \int_{1 / n}^{1-1 / n} H_{n}(t, s) d s\left(\nu_{n} \int_{\eta}^{1-1 / n}\left(\tau-\frac{1}{n}\right)\left(1-\frac{1}{n}-\tau\right) d \tau\right)^{-1} \geq M_{1},
\end{aligned}
$$

which implies that

$$
\left\|T_{n} u\right\| \geq\|u\| \text { for all } u \in \partial \Omega_{M_{1}} \cap K_{n} .
$$

In view of $\left(A_{2}\right)$, we can choose $R>M_{1}$ such that (3.4) holds. Hence, by Lemma 2.1, $T_{n}$ has a fixed point $u_{n} \in K_{n} \cap\left(\bar{\Omega}_{R} \backslash \Omega_{M_{1}}\right)$. By the same process as done in Theorem 1.1, the system (1.2) has a positive solution.

Example 3.2. Let

$$
f(t, y)=\frac{e^{\frac{1}{y}}}{t(1-t)}, \quad g(t, x)=\frac{e^{\frac{1}{x}}}{t(1-t)}
$$

and $\alpha=2, \eta=\frac{1}{3}$. Choose

$$
K(t)=L(t)=\frac{1}{t(1-t)}, \quad F(y)=e^{\frac{1}{y}}, \quad G(x)=e^{\frac{1}{x}} .
$$

Choose constant $M_{1}$ such that $M_{1} \leq \frac{4(n-3)}{n} e^{\frac{n}{1+6 n e^{n}}} \int_{1 / 3}^{1-1 / n}\left(s-\frac{1}{n}\right)\left(1-\frac{1}{n}-s\right) d s$. Then $\left(A_{1}\right),\left(A_{2}\right)$ and $\left(A_{4}\right)$ are satisfied. Hence, by Theorem 1.2 , system $(1.2)$ has a positive solution. 
Proof of Theorem 1.3. For $u \in \partial \Omega_{M_{2}} \cap K_{n}$, using (2.8), we have

$$
\begin{aligned}
\left(T_{n} u\right)(t) & =\frac{1}{n}+\int_{1 / n}^{1-1 / n} H_{n}(t, s) f\left(s,\left(B_{n} u\right)(s)\right) d s \\
& =\frac{1}{n}+\int_{1 / n}^{1-1 / n} H_{n}(t, s) f\left(s, \frac{1}{n}+\int_{1 / n}^{1-1 / n} H_{n}(s, \tau) g(\tau, u(\tau)) d \tau\right) d s .
\end{aligned}
$$

In view of $\left(A_{1}\right),\left(A_{5}\right)$ and Lemma 2.3 , we obtain

$$
\begin{aligned}
\leq & \frac{1}{n}+\int_{1 / n}^{1-1 / n} H_{n}(t, s) K(s) F\left(\frac{1}{n}+\int_{1 / n}^{1-1 / n} H_{n}(s, \tau) g(\tau, u(\tau)) d \tau\right) d s \\
\leq & \frac{1}{n}+\int_{1 / n}^{1-1 / n} H_{n}(t, s) K(s) F\left(\int_{1 / n}^{1-1 / n} H_{n}(s, \tau) g(\tau, u(\tau)) d \tau\right) d s \\
\leq & \frac{1}{n}+\int_{1 / n}^{1-1 / n} H_{n}(t, s) K(s) F\left(\int_{1 / n}^{1-1 / n} H_{n}(s, \tau) g\left(\tau, M_{2}\right) d \tau\right) d s \\
\leq & \frac{1}{n}+\int_{1 / n}^{1-1 / n} H_{n}(t, s) K(s) F\left(\int_{\eta}^{1-1 / n} H_{n}(s, \tau) g\left(\tau, M_{2}\right) d \tau\right) d s \\
\leq & \frac{1}{n}+\int_{1 / n}^{1-1 / n} H_{n}(t, s) K(s) F\left(\nu_{n} \int_{\eta}^{1-1 / n}\left(\tau-\frac{1}{n}\right)\left(1-\frac{1}{n}-\tau\right) g\left(\tau, M_{2}\right) d \tau\right) d s \\
= & \frac{1}{n}+F\left(\nu_{n} \int_{\eta}^{1-1 / n}\left(\tau-\frac{1}{n}\right)\left(1-\frac{1}{n}-\tau\right) g\left(\tau, M_{2}\right) d \tau\right) \int_{1 / n}^{1-1 / n} H_{n}(t, s) K(s) d s \\
\leq & \frac{1}{n}+\mu_{n} F\left(\nu_{n} \int_{\eta}^{1-1 / n}\left(\tau-\frac{1}{n}\right)\left(1-\frac{1}{n}-\tau\right) g\left(\tau, M_{2}\right) d \tau\right) \\
& \cdot \int_{1 / n}^{1-1 / n}\left(s-\frac{1}{n}\right)\left(1-\frac{1}{n}-s\right) K(s) d s \\
\leq & \frac{1}{n}+a \mu_{n} F\left(\nu_{n} \int_{\eta}^{1-1 / n}\left(\tau-\frac{1}{n}\right)\left(1-\frac{1}{n}-\tau\right) g\left(\tau, M_{2}\right) d \tau\right) \leq M_{2},
\end{aligned}
$$

which implies that

$$
\left\|T_{n} u\right\| \leq\|u\| \text { for all } u \in \partial \Omega_{M_{2}} \cap K_{n} .
$$

By $\left(A_{3}\right)$, we can choose $\rho \in\left(0, M_{2}\right)$ such that (3.7) holds. Hence, $T_{n}$ has a fixed point $u_{n} \in K_{n} \cap\left(\bar{\Omega}_{M_{2}} \backslash \Omega_{\rho}\right)$. By the same process as done in Theorem 1.1, the system (1.2) has a positive solution.

Example 3.3. Let

$$
f(t, y)=\left\{\begin{array}{ll}
\frac{y e^{\frac{1}{y}}}{t(1-t)}, & y \leq 1, \\
\frac{e}{t(1-t)}, & y>1,
\end{array} \quad g(t, x)= \begin{cases}\frac{x e^{\frac{1}{x}}}{t(1-t)}, & x \leq 1 \\
\frac{e}{t(1-t)}, & x>1\end{cases}\right.
$$


and $\alpha=2, \eta=\frac{1}{3}$. Choose

$$
K(t)=L(t)=\frac{1}{t(1-t)}, \quad F(y)=\left\{\begin{array}{ll}
y e^{\frac{1}{y}}, & y \leq 1, \\
e, & y>1,
\end{array} \quad G(x)= \begin{cases}x e^{\frac{1}{x}}, & x \leq 1 \\
e, & x>1\end{cases}\right.
$$

and $\beta_{1}=\beta_{2}=1$. Choose constant $M_{2}$ such that

$$
M_{2} \geq \max \left\{1, \frac{1}{n}+6 F\left(e(1-3 / n) \int_{1 / 3}^{1-1 / n} \frac{(s-1 / n)(1-1 / n-s)}{s(1-s)} d s\right)\right\} .
$$

Then $\left(A_{1}\right),\left(A_{3}\right)$ and $\left(A_{5}\right)$ are satisfied. Hence, by Theorem 1.3, system (1.2) has a positive solution.

Proof of Theorem 1.4. By $\left(A_{1}\right)$ and $\left(A_{4}\right)$, we obtain $(3.10)$. By $\left(A_{5}\right)$ we can choose a constant $M_{2}>M_{1}$ such that (3.11) holds. Then $T_{n}$ has a fixed point $u_{n} \in K_{n} \cap\left(\bar{\Omega}_{M_{2}} \backslash \Omega_{M_{1}}\right)$. By the same process as done in Theorem 1.1, the system (1.2) has a positive solution.

Example 3.4. Let

$$
f(t, y)=\frac{1}{t(1-t)} \frac{1}{\sqrt{y}}, \quad g(t, x)=\frac{1}{t(1-t)} \frac{1}{x^{2}}
$$

and $\alpha=2, \eta=\frac{1}{3}$. Choose

$$
K(t)=L(t)=\frac{1}{t(1-t)}, \quad F(y)=\frac{1}{\sqrt{y}}, \quad G(x)=\frac{1}{x^{2}} .
$$

Choose constants $M_{1}$ and $M_{2}$ such that $M_{1} \leq \frac{4(n-3)}{\sqrt{n\left(6 n^{3}+1\right)}} \int_{1 / 3}^{1-\frac{1}{n}}\left(s-\frac{1}{n}\right)\left(1-\frac{1}{n}-\right.$ $s) d s$ and $M_{2} \geq \frac{1}{6 n}\left(\frac{1}{6}-\sqrt{\frac{n}{n-3}}\left(\int_{1 / 3}^{1-1 / n} \frac{(s-1 / n)(1-1 / n-s)}{s(1-s)} d s\right)^{-1 / 2}\right)^{-1}$. Then $\left(A_{1}\right)$, $\left(A_{4}\right)$ and $\left(A_{5}\right)$ are satisfied. Hence, by Theorem 1.4 , system (1.2) has a positive solution.

\section{References}

[1] R. P. Agarwal and D. O'Regan, Singular Differential and Integral Equations with Applications, Kluwer Academic Publishers, Dordrecht, 2003.

[2] A. V. Bicadze and A. A. Samarskiř, Some elementary generalizations of linear elliptic boundary value problems, Dokl. Akad. Nauk SSSR 185 (1969), 739-740.

[3] A. V. Bitsadze, On the theory of nonlocal boundary value problems, Dokl. Akad. Nauk SSSR 277 (1984), no. 1, 17-19.

[4] - A class of conditionally solvable nonlocal boundary value problems for harmonic functions, Dokl. Akad. Nauk SSSR 280 (1985), no. 3, 521-524.

[5] L. E. Bobisud, Existence of solutions for nonlinear singular boundary value problems, Appl. Anal. 35 (1990), no. 1-4, 43-57.

[6] W. Cheung and P. Wong, Fixed-sign solutions for a system of singular focal boundary value problems, J. Math. Anal. Appl. 329 (2007), no. 2, 851-869.

[7] R. Dalmasso, Existence and uniqueness of positive radial solutions for the Lane-Emden system, Nonlinear Anal. 57 (2004), no. 3, 341-348.

[8] D. Guo and V. Lakshmikantham, Nonlinear Problems in Abstract Cones, Academic Press, Inc., Boston, MA, 1988. 
[9] C. P. Gupta, Solvability of a three-point nonlinear boundary value problem for a second order ordinary differential equation, J. Math. Anal. Appl. 168 (1992), no. 2, 540-551.

[10] V. A. Il'in and E. I. Moiseev, A nonlocal boundary value problem of the first kind for the Sturm-Liouville operator in differential and difference interpretations, Differentsial'nye Uravneniya 23 (1987), no. 7, 1198-1207.

[11] _ A nonlocal boundary value problem of the second kind for the Sturm-Liouville operator, Differentsial'nye Uravneniya 23 (1987), no. 8, 1422-1431, 1471.

[12] P. Kang and Z. Wei, Three positive solutions of singular nonlocal boundary value problems for systems of nonlinear second-order ordinary differential equations, Nonlinear Anal. 70 (2009), no. 1, 444-451.

[13] P. Kelevedjiev, Nonnegative solutions to some singular second-order boundary value problems, Nonlinear Anal. 36 (1999), no. 4, Ser. A: Theory Methods, 481-494.

[14] B. Liu, Positive solutions of a nonlinear three-point boundary value problem, Comput. Math. Appl. 44 (2002), no. 1-2, 201-211.

[15] B. Liu, L. Liu, and Y. Wu, Positive solutions for singular second order three-point boundary value problems, Nonlinear Anal. 66 (2007), no. 12, 2756-2766.

[16] _ Positive solutions for singular systems of three-point boundary value problems, Comput. Math. Appl. 53 (2007), no. 9, 1429-1438.

[17] R. Ma, Positive solutions of a nonlinear three-point boundary-value problem, Electron. J. Differential Equations 1999 (1999), no. 34, 8 pp.

[18] M. Moshinsky, Sobre los problems de conditions a la frontiera en una dimension de caracteristicas discontinuas, Bol. Soc. Mat. Mexicana 7 (1950), 1-25.

[19] T. Timoshenko, Theory of Elastic Theory, McGraw-Hill, New York, 1971.

[20] J. R. L. Webb, Positive solutions of some three point boundary value problems via fixed point index theory, Nonlinear Anal. 47 (2001), no. 7, 4319-4332.

[21] S. Xie and J. Zhu, Positive solutions of boundary value problems for system of nonlinear fourth-order differential equations, Bound. Value Probl. 2007 (2007), Art. ID 76493, 12 pp.

[22] Z. Zhao, Solutions and Green's functions for some linear second-order three-point boundary value problems, Comput. Math. Appl. 56 (2008), no. 1, 104-113.

[23] Y. Zhou and Y. Xu, Positive solutions of three-point boundary value problems for systems of nonlinear second order ordinary differential equations, J. Math. Anal. Appl. $\mathbf{3 2 0}$ (2006), no. 2, 578-590.

NASEer AhMAd ASIF

Centre for Advanced Mathematics and Physics

National University of Sciences And TeChnology

Campus of College of Electrical and Mechanical Engineering

Peshawar Road, Rawalpindi, Pakistan

E-mail address: naseerasif@yahoo.com

Paul W. Eloe

Department of Mathematics

UNIVERSITY OF DAYTON

DAYTON, OHIO 454-2316, USA

E-mail address: Paul.Eloe@notes.udayton.edu

Rahmat Ali KHAN

Centre for Advanced Mathematics and Physics

National University of Sciences and TeChnology

Campus of College of Electrical and Mechanical Engineering

Peshawar Road, Rawalpindi, Pakistan 
AND

Department of Mathematics

UNIVERSITY OF DAYTON

DAYTON, OHIO 45469-2316, USA

E-mail address: rahmat_alipk@yahoo.com 